\title{
Knowledge, Attitudes and Practices regarding Long-Term Complications of COVID-19 among a Sub-Urban Population in Sri Lanka
}

1. Chamindri Witharana, Faculty of Medicine, University of Colombo, Sri Lanka

2. Kithmini Siridewa, Faculty of Medicine, University of Colombo, Sri Lanka

3. Manuj Weerasinghe, Faculty of Medicine, University of Colombo, Sri Lanka

4. Indika Karunathilake, Faculty of Medicine, University of Colombo, Sri Lanka

Corresponding author: Dr. Chamindri Witharana, Department of Biochemistry and Molecular Biology, Faculty of Medicine, University of Colombo, Sri Lanka

E-mail: Chamindri@bmb.cmb.ac.lk

\begin{abstract}
Background

Recently, a surge of COVID 19 was observed globally, regionally and nationally. With increasing numbers of cases, the frequency of long COVID is on the rise. Management and control of long COVID depends on changes in respect of human behaviors and requires understanding of knowledge, attitudes, and practices (KAP) regarding health threats.
\end{abstract}

\section{Methods}

A descriptive cross sectional study using online survey to gather data on the socio-economic background, knowledge, attitudes and practices on long-term complications of COVID.

\section{Results}

Out of 201 respondents, $89.2 \%$ participants have heard about long-term complications of COVID 19. Only $35.9 \%$ have demonstrated the adequate knowledge in the questions relating 
to co-morbidities and risk factors of COVID-19. A total of $92.2 \%$ believe that they should adhere to preventive measures following vaccination. Less than $60 \%$ were following the advices on avoiding unnecessary travel and crowded places. Further, less than $50 \%$ were following COVID preventive measures.

\section{Conclusion}

Although the majority of participants have heard about long term complications and common symptoms, the knowledge regarding co-morbidities that can lead to severe disease and long COVID was not satisfactory. The attitudes of the participants indicated increasing concern about long COVID. Practices indicate lack of adherence to key measures such as avoiding crowded places. These findings highlight the need for further increasing of awareness. 
Keywords: long COVID, COVID 19 vaccination, COVID awareness

\section{Background}

The first outbreak of COVID-19 was identified in December 2019 from Wuhan City in Hubei Province of China and in January 2020 the World Health Organization (WHO) declared it as a Public Health Emergency of International Concern. The first case of COVID-19 in South Asia was identified in January 2020, and in March 2020 COVID-19 was declared a pandemic by WHO. Sri Lanka reported it's first case on $28^{\text {th }}$ January 2020 (1).

The COVID-19 disease is caused by the SARS-CoV-2 virus which has given rise to several variants in the recent months. The latest variants are found to be spreading faster and has given rise to the increased number positive cases and mortality rates in the recent months (2). This is evident with the global statistics whereas at the first week of September 2021, almost 220 million cumulative cases have been recorded, with 4.5 million deaths worldwide (1). As of the first week of October, Sri Lanka has recorded a total of 517882 COVID-19 positive cases with a mortality burden of 12907 deaths (3).

As with other respiratory illnesses, infection with SARS-CoV-2 virus can cause mild symptoms including a runny nose, sore throat, cough, and fever. It can be more severe for some persons and can lead to pneumonia. In other cases, the disease can be fatal, especially in older people, and those with pre-existing medical conditions who are at higher risk of getting complications $(4,5)$.

The incubation period is from 2 to 14 days with an average of 5 days. Most patients recover within two weeks whereas the progress of those with severe complications can take longer 
time with poor prognosis. After recovery, some patients may experience post-COVID symptoms. These symptoms are multi-system and can range from mild to moderate to severe and debilitating. This phenomenon is generally known as "long COVID" $(5,6)$.

The exact mechanism of long COVID is yet to be understood. However the occurrence and severety of post-COVID symptoms are related to multiple factors such as virulance of the virus, host immune response, severity of the disease, vaccination status, existing comorbidities and management of the initial infections (5).

In order to design effective managegement and and control strategies of long COVID, there should be adequate information on the knowledge, attitudes and practices of people. Research have identified that age, educational level and other socioeconomic factors amongst many others have notable impact on the knowledge, attitudes and practices regarding COVID-19. Even though several studies have been carried out on preventive and control measures of COVID 19, there is a paucity of evidence regarding long COVID (7,8). (Azlan et al.,Alhazmi et al., 2020; Noreen et al., 2020).

In light of this limited information regarding long COVID, we assessed the awareness of the long-term health effects of COVID-19, and Knowledge, Attitudes and Practices regarding COVID-19 among the general public in a cross-sectional online study carried out within a two month period. The findings of this study shall be beneficial in order to inform the design of effective managegement and and control strategies for long COVID. Futher the finidngs will be helpful in evaluation of the effectiveness of the COVID 19 vaccination.

\section{Methods}




\section{Aim, design and setting of the study}

An online survey of a pre-determined questionnaire was used to gather data on the socioeconomic background, knowledge, attitudes and practices on long-term complications of COVID. The questionnaire was administered as a google form, and shared on social media platforms Whatsapp and Facebook and participants who were living in Colombo district were invited to participate. In addition, telephone conversation was used for participants who did not have access to internet facilities The online questionnaire was available from early August to mid-September 2021.

A brief description of the investigators, study questionnaire, purpose of study, study duration and participant responsibilities, risks and benefits, and confidentiality was given before filling the questionnaire. The participation was entirely voluntary and anyone could withdraw from the study at any time if they were not willing to proceed further. Ethical clearence for the study was obtained from the Ethical review committee, Faculty of Medicine, University of Colombo, Sri Lanka.

\section{Questionaire Design Process}

The first section of the questionnaire constisted of items on socio-demographics, such as age, ethnicity, level of education, gender, employment status and economic level.

The rest of the questionnaire was divided into five parts which assessed the following: (1) knowledge about COVID-19 (1.1: co-morbidities and risk factors, 1.2: complications, and 1.3: long COVID symptoms), (2) Attitudes on COVID 19, (3) Practices with regard to COVID 19, (4) any personal experience with COVID-19 diagnosis or quarantine during the past 3 months, and (5) vaccination history of participant. 
Questions related to knowledge had three options, “yes / no / don't know”. There was a total of fourty-eight knowledge questions, and for each item, a score of 1 was given for 'yes' and a score of 0 for 'no' and 'don't know'. An individual score of less than 40 percentage (40\%) was taken as inadequate, while the score of $40 \%$ or higher was counted adequate.

For the ten questions related to attitudes, there were five options ranging from strongly disagree to strongly agree. A descriptive approach was employed in examining this data. There were fifteen practice questions, each of which had five options which ranged from never to always. The individual scores ranged as 0 for never, 1 for rarely, 2 for sometimes, 3 for frequently and 4 for always. An overall score of $40 \%$ or higher were as taken as adequate.

\section{Statistical Analysis}

The descriptive analysis of percentages (\%) was used to present demographic characteristics regarding the level and distribution of knowledge, attitude and practice toward COVID 19. Non-parametric tests were used to explore the association between demographic characteristics variables and COVID-19 knowledge scores. All data were analyzed using Microsoft Excel 2010 and SPSS 20.0, with $\mathrm{P}<0.05$ considered statistically significant.

\section{Results}

\section{Demographic characteristics}

The results of the online questionnaire turned out an overall response from 201 respondents, of which 92 filled the questionnaire in English language and 105 respondents filled it in Sinhala language. The participants comprise a majority of females than male participants with a numbers of $52.7 \%$ and $47.2 \%$ respectively. 
Majority of the participants have answered most of the questions with a high response rate. The highest number of $22.3 \%$ of respondents is of the less than 25 years group. This is closely followed by $21.8 \%$ in the age group $26-35$ years, $16.5 \%$ in the $46-55$ years group, $16.1 \%$ in $36-45$ years, $12.9 \%$ in the $56-65$ years group and lastly $10.4 \%$ in the 66 years and above group.

\section{Knowledge}

The results of this survey reveal that $89.2 \%$ participants have heard about Long term complications of COVID 19. This comprises of different information sources, with the highest of 59.5\% from television, $32.4 \%$ from internet, $5.7 \%$ from health care workers and only $2.9 \%$ from Newspapers / Magazines.

The responses of $26.2 \%$ of respondents have indicated that they do not know the answers to at least 10 of the total of 48 questions of the knowledge section. Only $35.9 \%$ have demonstrated the adequate knowledge in the questions relating to co-morbidities and risk factors of COVID-19. Over $60 \%$ were aware of common symptoms of long COVID such as breathlessness, cough, joint pains, muscle aches, headache, fatigue and feeling weak. Less than $20 \%$ were aware of symptoms such as insomnia, nightmares, weight changes, skin rashes (Figure 1-3). 
i)

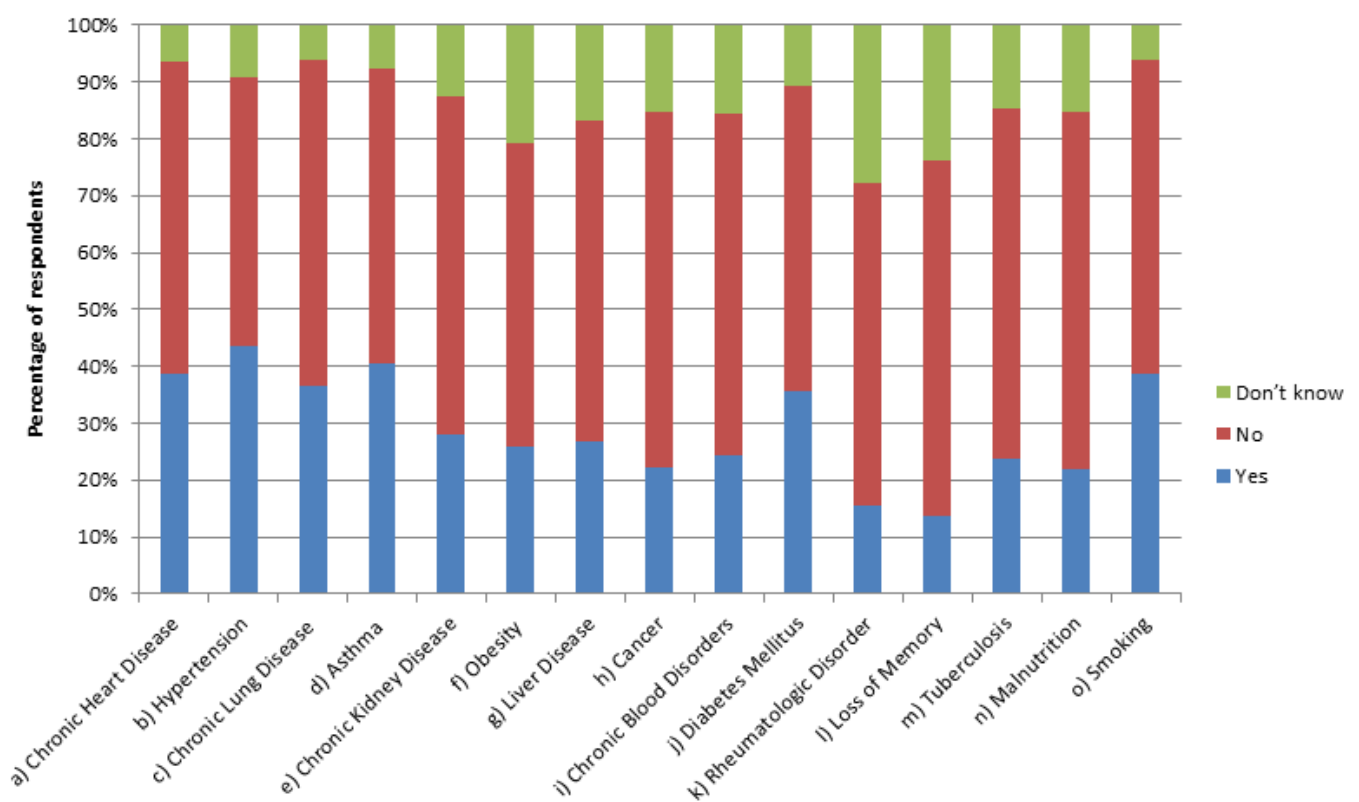

Figure 1: Knowledge regarding co-morbidities and risk factors of COVID-19

ii)

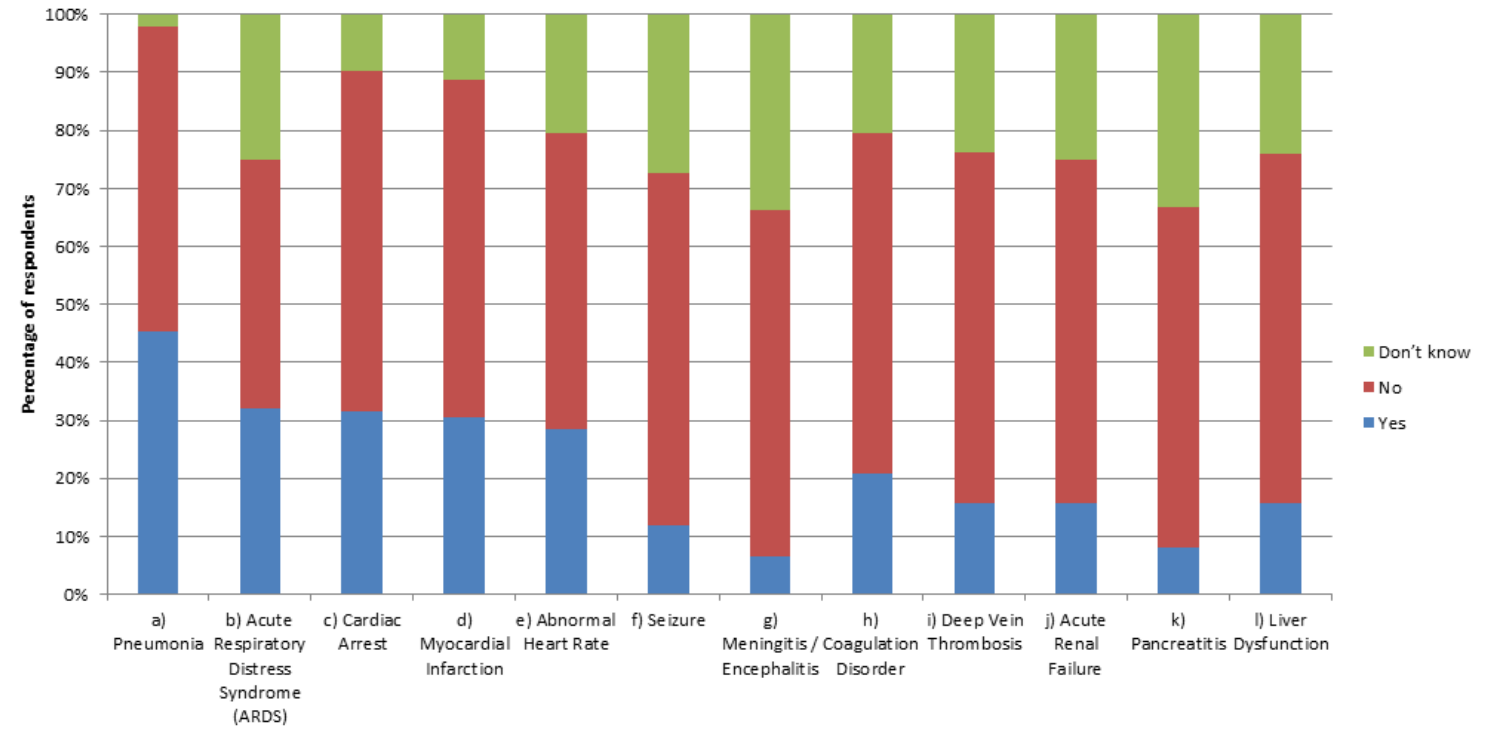

Figure 2: Knowledge regarding complications of COVID-19 


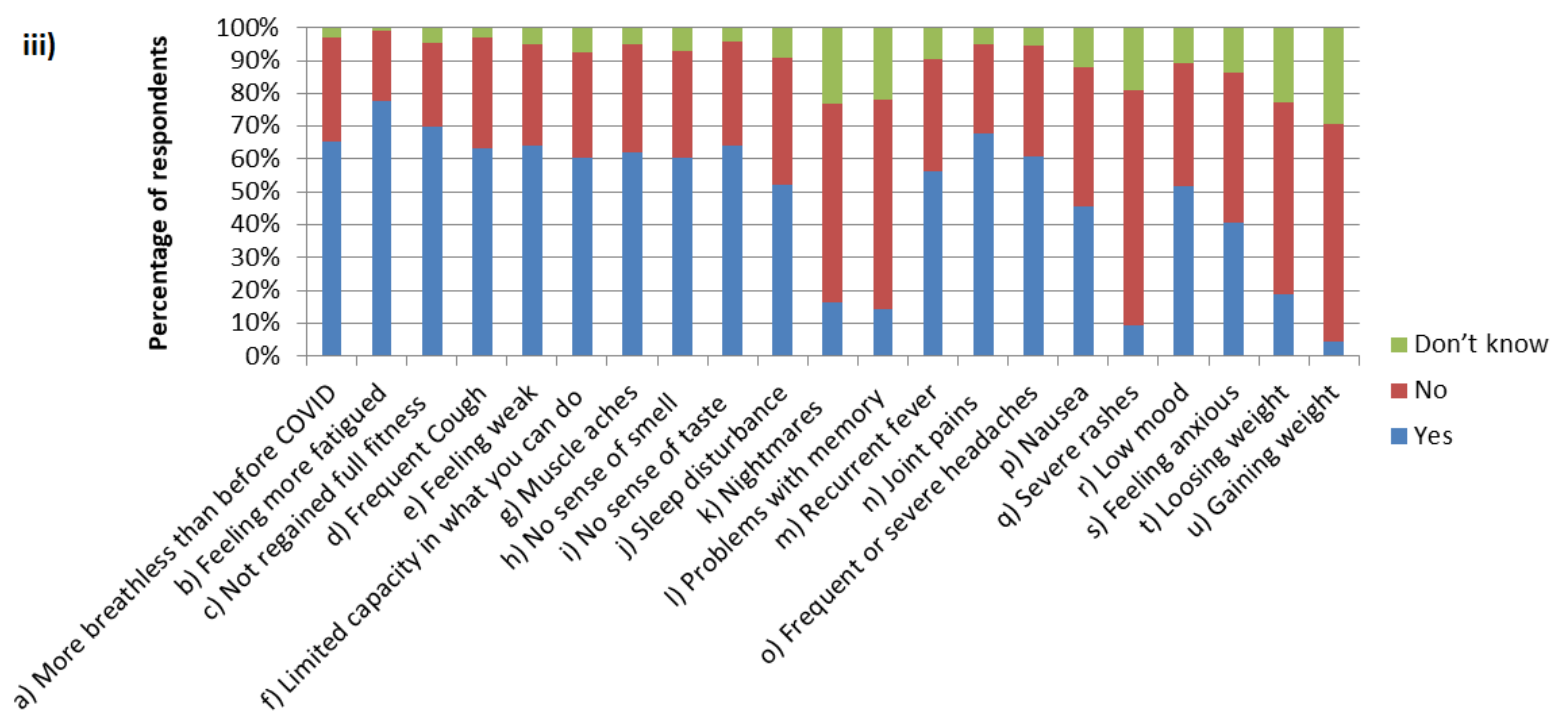

Figure 3: Knowledge regarding long COVID symptoms

\section{Attitude}

Majority of the respondents believed that covid-19 should be taken into serious consideration, with $15.8 \%$ agreeing and $73.9 \%$ strongly agreeing with the statement. A total of $92.2 \%$ agrees that they believe that they should adhere to preventive measures following vaccination.

Most (85\%) of the respondents believe that people who are sick with COVID-19 must not be afraid to tell others that they have the disease. Over $80 \%$ believe that it is embarrassing to have COVID-19. However, less than 5\% feel that people who are sick with covid-19 should be rejected by society.

The majority of respondents (72\%) feel they are vulnerable to COVID 19. A cumulative majority of $84.5 \%$ are concerned about getting infected with COVID-19. 


\section{Practice}

Over $60 \%$ stated that they get medical advice when not feeling well. Nearly $90 \%$ wear a mask when they go out. Less than $60 \%$ were following the advices on avoiding unnessary travel and crowded places. Further, less than $50 \%$ were following instructions on performing hand hygiene immediately after discarding a mask, keeping a distance of $1 \mathrm{~m}$ or more between each other when stayisng outside and regularly cleaning hands with alcohol-based hand rub/ wash with soap and water (Figure 4). 


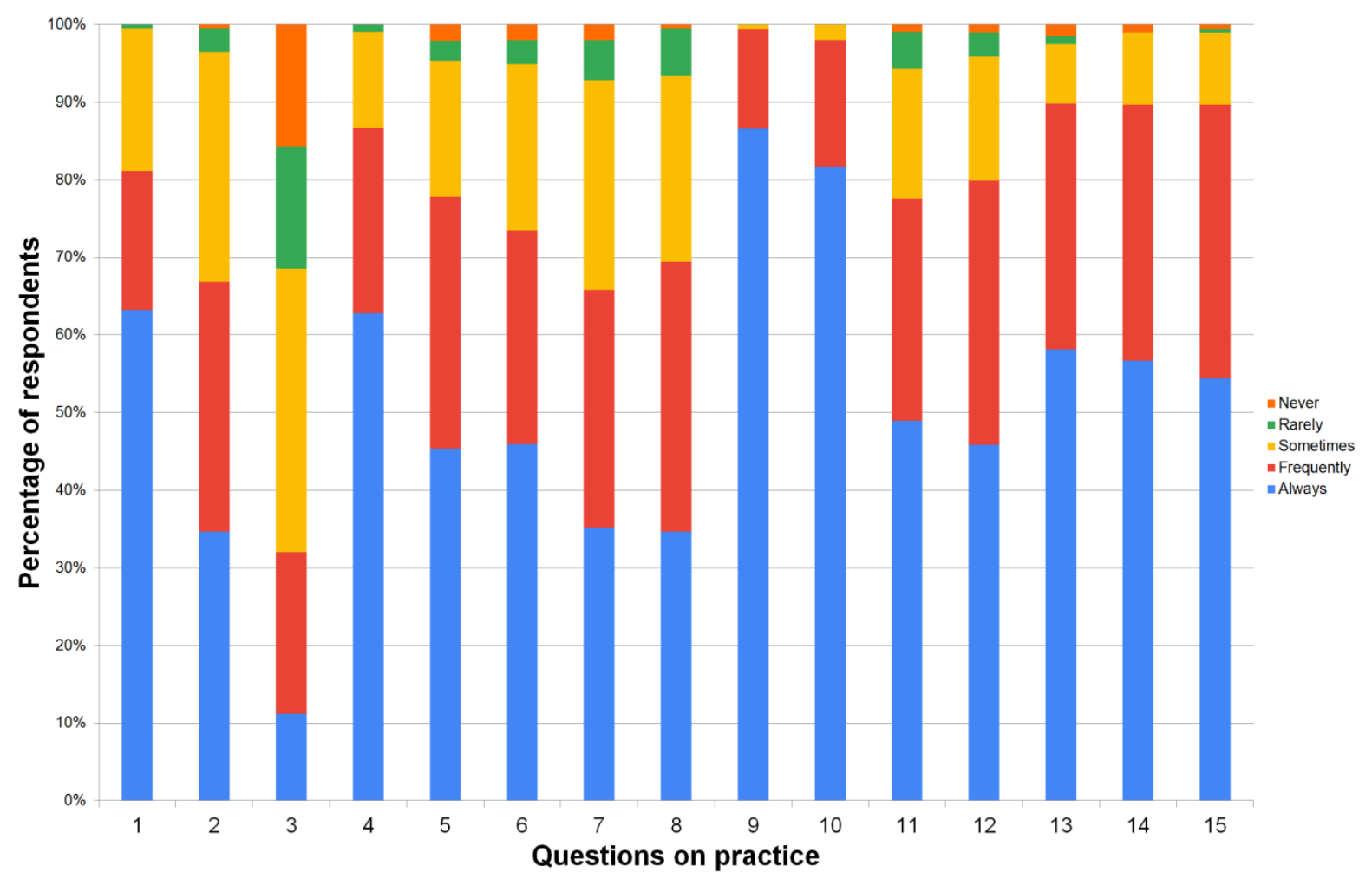

Figure 4 - Responses for questions on practices

\begin{tabular}{|c|l|c|l|}
\hline 1 & Getting medical advice when not feeling well & 9 & Wearing a mask whenever going outside. \\
\hline 2 & Maintain healthy eating & 10 & Ensuring that mouth and nose are covered \\
properly
\end{tabular}




\section{Discussion}

Although our results reveal that the greater majority of participants had heard about the complications of COVID-19, the results conversely demonstrate a lack of knowledge about the modes of transmission and clinical manifestations of COVID19. These further include the lack of knowledge of co-morbidities and risk factors such as diabetes, obesity, chronic heart or lung disease, hypertension and asthma can lead to severe disease if infected. This raises a concern regading the effectvieness of health education conveyed by elctronic media. However it should be noted that a greater majority had responded adequately for the knowledge questions relating to the complications of COVID-19 ${ }^{9}$.

The knowledge on long COVID symptoms is an area that is not much known among the respondents with just over half displaying adequate knowledge. This may be due to the short duration of the pandemic, past one and a half years, and people are not aware of the longterm consequences such as insomnia, nightmares, weight changes, skin rashes. However, they are potentially debilitating symptoms that would need to be addressed and educated among the general public.

The attitudes of participants show a combination of favourable responses, with majority agreeing on common principles in order to increase awareness of COVID situations. However, the specific responses relating to perceptions of the society towards those infected with the disease should be monitored closely so that the individuals and society do not suffer detrimental emotional situations. 
The varable levels of adherence, specially related to social distancing and frequent hand washing or disinfecting hands, which are principal methods of prevention of COVID-19 raises serious concerns. ${ }^{10,11}$

Sri Lanka has been under the control of several lockdown and islandwide curfew periods since COVID-19 early 2020. Although there are many programmes carried out to educate the general public, the success of such actions is still in question ${ }^{9}$. The knowledge, attitudes and practices of people regarding a disease concern greatly vary within a society ${ }^{12}$.

The present study comprises several limitations, with the relatively small sample size and the responses mainly based on social media usage. Furthermore, the majority of respondents had been infected with Covid-19 and undergone treatment, thus the group does not adequately represent those who have not had that experience. Despite the limitations of present study, including the short study time period, the results brings insight on the knowledge of long COVID symptoms and prevalent knowledge, attitudes and practices regarding COVID-19 amongst a study sample of residents in Colombo, Sri Lanka.

\section{Conclusion}

The study has identified gaps in the the knowledge of long COVID symptoms and prevalent knowledge, attitudes and practices regarding COVID-19 and long COVID. The identified needs further attention as a matter of urgency in the fight against COVID-19, specailly in the light of current global surge of COVID 19 cases and emergence of new varints. Futher, the success of COVID 19 vaccination programme depends on the adherecne to preventive measures by general public. A larger study extending beyond the Colombo district will help 
to understand the specific issues pertaining to different geographical and population segments and will contribute towards controlling of of Covid 19 in Sri Lanka and globally.

\section{Declarations}

Ethics approval and consent to participate: This project has been approved by the Ethics Review Committee, Faculty of Medicine, University of Colombo.

Consent for publication: Not applicable

Availability of data and materials: The datasets used and/or analysed during the current study are available from the corresponding author on reasonable request.

Competing interests: The authors declare that they have no competing interests

Funding: Not applicable

Authors' contributions: IK and CW proposed the research idea. IK and MJ developed the tool. Data collection was carried out by $\mathrm{CW}$ and KS. Data analysis by CW and IK. All authors contributed to writing of the manuscript. All authors read and approved the final manuscript.

Acknowledgements: Not applicable 


\section{References}

1. WHO. Timeline: WHO's COVID-19, ,https://www.who.int/emergencies/diseases/novelcoronavirus-2019/interactive-timeline, 2021. Accessed on 17 September 2021

2. Center for Disease Control and Prevention . Delta Variant: What We Know About the Science, 2021 <https://www.cdc.gov/coronavirus/2019-ncov/variants/delta-variant.html>, Accessed on 17 September 2021

3. Epidemiological Unit. Ministry of Health. (a), Report on present situation- Global, <http://www.epid.gov.lk/web/images/pdf/corona_virus_report/sitrep-gl-en-15-

09_10_21.pdf>, 2021. Accessed on 17 September 2021

4. Baradaran A, Ebrahimzadeh $\mathrm{MH}$, Baradaran A, Kachooei AR. Prevalence of Comorbidities in COVID-19 Patients: A Systematic Review and Meta-Analysis. Arch Bone Jt Surg. 2020;8(Suppl 1):247-255. doi:10.22038/abjs.2020.47754.2346

5. Aiyegbusi OL, Hughes SE, Turner G, et al. Symptoms, complications and management of long COVID: a review. Journal of the Royal Society of Medicine. 2021;114(9):428-442. doi:10.1177/0141076821103285

6. WHO. Statement for Healthcare Professionals, https://www.who.int/news/item/11-062021-statement-for-healthcare-professionals-how-covid-19-vaccines-are-regulated-for-safetyand-effectiveness, 2021. Accessed on 18 September 2021 
7. Azlan AA, Hamzah MR, Sern TJ, Ayub SH, Mohamad E. Public knowledge, attitudes and practices towards COVID-19: A cross-sectional study in Malaysia. PLoS One. 2020;15(5):e0233668. Published 2020 May 21. doi:10.1371/journal.pone.0233668

8. Afzal MS, Khan A, Qureshi UUR, et al. Community-Based Assessment of Knowledge, Attitude, Practices and Risk Factors Regarding COVID-19 Among Pakistanis Residents During a Recent Outbreak: A Cross-Sectional Survey. J Community Health. 2021;46(3):476486. doi:10.1007/s10900-020-00875-Z

9. Erandi KK, Mahasinghe AC, Perera SS, Jayasinghe S. Effectiveness of the strategies implemented in Sri Lanka for controlling the COVID-19 outbreak. Journal of Applied Mathematics. 2020 Jan 1;2020.

10. Kusuma D, Pradeepa R, Khawaja KI, et al. Low uptake of COVID-19 prevention behaviours and high socioeconomic impact of lockdown measures in South Asia: Evidence from a large-scale multi-country surveillance programme. SSM Popul Health. 2021;13:100751. Published 2021 Feb 13. doi:10.1016/j.ssmph.2021.100751

11. Yang Chan EY, Shahzada TS, Sham TST, et al. Narrative review of non-pharmaceutical behavioural measures for the prevention of COVID-19 (SARS-CoV-2) based on the HealthEDRM framework. Br Med Bull. 2020;136(1):46-87. doi:10.1093/bmb/ldaa030

12. Matthias AT, Padmasiri MS, Dharani UG. Knowledge, Attitudes, and Practices on COVID-19 Among Medical Students in Sri Lanka. Asia Pacific Journal of Public Health. 2021 Feb 9:1010539521993663. 